\title{
HARMONIC MAPS OF DEGREE 1 INTO THE UNIT 2-SPHERE*
}

\author{
YUXIN GE ${ }^{\dagger}$
}

1. Introduction. Let $S^{2}$ be the unit 2-sphere in $\mathbb{R}^{3}$ and $M$ a closed Riemann surface of genus greater than 1 . Let $H^{1}\left(M, S^{2}\right)$ be the set of all $u \in H^{1}\left(M, \mathbb{R}^{3}\right)$ with $u(x) \in S^{2}$ for a.e. $x \in M$. The Dirichlet energy functional $E$ on $H^{1}\left(M, S^{2}\right)$ is given by

$$
E(u)=\frac{1}{2} \int_{M}|\nabla v|^{2} d V o l_{M}, \quad \forall u \in H^{1}\left(M, S^{2}\right)
$$

The critical points of the energy functional are harmonic maps, which satisfy the following Euler-Lagrange equation

$$
\triangle u+|\nabla u|^{2} u=0
$$

where $\triangle$ is the Laplace-Beltrami operator. Notice that the energy functional does not depend on the choice of the metrics on $M$, provided that they are compatible with the complex structure on $M$. So the weak harmonic maps from $M$ to $S^{2}$ depend only on the complex structure on $M$. In this paper, we will consider the following problem: is there a harmonic map of degree 1 from $M$ to $S^{2}$ ? There is definitely no $E$-minimum, since it would be holomorphic or anti-holomorphic [14]. And it is well known that there is no harmonic map of degree 1 from a torus to a 2 -sphere (see [8]). In 1978, using a minimization procedure in a suitable space, L. Lemaire [14] obtained that there exists a harmonic map of degree one from $M$ to $S^{2}$ provided that $M$ has three planes of symmetry. Using the result due to J. Jost [13], G.F. Wang [21] improved this result under the condition that $M$ has one plane of symmetry. Here, we will follow a strategy proposed by J.M. Coron [5]; using the Minimax Principle of J. Jost; to prove the existence of such a harmonic map in a different class of Riemann surfaces defined as below. In particular, we do not need any symmetry assumption and our existence result proves the existence of degree 1 harmonic map on all Riemann surfaces described by an open subset of the moduli space of Riemann surfaces. Let $R_{1}$ and $R_{2}$ be two closed Riemann surfaces with positive genus $g_{1}$ and $g_{2}$ respectively. For each $j$, fix a point $p_{j} \in R_{j}$, and a coordinate neighborhood $\left(V_{j}, z_{j}\right)$ around $p_{j}$ such that $z_{j}\left(p_{j}\right)=0$ and $z_{j}\left(V_{j}\right)=B=\left\{z_{j} \in \mathbb{C}:\left|z_{j}\right|<2\right\}$. For every complex $\epsilon_{j}$, with $0<\left|\epsilon_{j}\right|<1$, we set

$$
V_{j, \epsilon_{j}}=z_{j}^{-1}\left(\left\{z_{j} \in \mathbb{C}:\left|\epsilon_{j}\right| \leq\left|z_{j}\right| \leq 1\right\}\right), \quad j=1,2 .
$$

Now, fix two points $q_{1}$ and $q_{2} \in S^{2}$, and two coordinate neighborhoods $\left(U_{j}^{\prime}, z_{j}^{\prime}\right)$ around $q_{j}$ such that $z_{j}^{\prime}\left(q_{j}\right)=0$ and $z_{j}^{\prime}\left(U_{j}^{\prime}\right)=B(0,1)=\left\{z_{j}^{\prime} \in \mathbb{C}:\left|z_{j}^{\prime}\right|<1\right\}$. We define

$$
U_{j, \epsilon_{j}}^{\prime}=U_{j}^{\prime}-\left(z_{j}^{\prime}\right)^{-1}\left(\left\{z_{j}^{\prime} \in \mathbb{C}:\left|z_{j}^{\prime}\right| \leq\left|\epsilon_{j}\right|\right\}\right), \quad j=1,2 .
$$

Then, identifying $U_{j, \epsilon_{j}}^{\prime}$ and $V_{j, \epsilon_{j}}$ for $j=1,2$ by the mappings

$$
z_{j}^{\prime} \cdot z_{j}=\epsilon_{j}
$$

${ }^{*}$ Received September 22, 1998; accepted for publication April 2, 1999.

†C.M.L.A., E.N.S de Cachan, 61, avenue du Président Wilson, 94235 Cachan Cedex, France and Département de Mathématiques, Faculté de Sciences et Technologie, Université Paris XII-Val de Marne, 61, avenue du Général de Gaulle, 94010 Créteil Cedex, France (ge@cmla.ens-cachan.fr). 
we obtain a Riemann surface $R_{\epsilon_{1}, \epsilon_{2}}$ of genus $g=g_{1}+g_{2}$ with $g \geq 2$. In fact, these surfaces $R_{\epsilon_{1}, \epsilon_{2}}$ with specific conformal structure are close to the infinity in $T_{g}$, the Teichmüller space of genus $g \geq 2$. Therefore, we obtain the following theorem

THEOREM 1. There exists $\alpha>0$ such that if $\left|\epsilon_{j}\right|<\alpha$ for $j=1,2$, then we can find a harmonic map of degree 1 from $R_{\epsilon_{1}, \epsilon_{2}}$ to $S^{2}$.

More generally, with the help of Schiffer's interior variation [12] (Appendix A), we establish our main result

THEOREM 1'. There exists an open subset $H$ in $T_{g}$ such that for each Riemann surface $R \in H$, we can find a harmonic map of degree 1 from $R$ to $S^{2}$.

Here, our method follows those in [4] and in [9]. For this type of problems, the minimizing method fails. But the energy level sets near the minimum have a nontrivial topology, which allows us to look for a critical point with the help of a topological method. Our approach is the following. In Section 2, we will study minimizing sequences. We observe that concentration phenomena occur. In some way, our analysis is similar to P.L. Lions' concentration compactness results [15] on the best constant of Sobolev embedding for the limiting case, and to results of BrezisCoron [2] and Struwe [19] on $H$-surfaces. More generally, C.Y. Wang [20] (see also [16]) showed that every Palais-Smale sequence may concentrate its energy at finitely many points where the sequence may generate a nontrivial harmonic mapping from $S^{2}$ to $S^{2}$ (called a bubble). This is the reason why Sacks-Uhlenbeck [17] and K. Uhlenbeck [23] studied a family of perturbed functionals and established a perturbed Morse theory for harmonic maps. M. Struwe [18] developped a similar theory using the heat flow for harmonic maps (see also K.C. Chang [3]) and J. Jost [13] carried out the bubbling process of a mini-max value for the Dirichlet energy of a mapping from a surface to a closed Riemannian manifold, a crucial result in our proof.

In Section 3, we will analyze nonconstant harmonic maps from Riemann surfaces to the unit 2-sphere $S^{2}$, based on Schiffer's interior variation in Teichmüller space. Notice that $E$ and harmonic maps depend only on the conformal structure of surfaces; we can consider the above problem on the set of all biholomorphic equivalence classes of closed Riemann surfaces of genus $g$, denoted by $\mathcal{M}_{g}$ (the Riemann's moduli space of genus $g$ ). Actually, we construct a family $F$ of Riemann surfaces of genus $>1$ containing the subfamily $A=\left\{R_{\epsilon_{1}, \epsilon_{2}}:\left|\epsilon_{i}\right|<1, i=1,2\right\}$ and we show that the energy of nonconstant harmonic maps from each Riemann surface to $S^{2}$ is uniformly bounded from below.

In the last section, we construct a non-trivial loop in an energy level set near the minimum, which is contractible in higher level sets. Then, thanks to a result of J. Jost [13], based on ideas of Sacks-Uhlenbeck [17], we can apply a topological critical point theory in a level set of the energy functional where no blow-up can occur. In particular the result of Section 3 shows that no bubbling effect occurs, and this establishes our result.

2. Study of a minimizing sequence. First, we can label homotopy classes as the following connected components

$$
\begin{aligned}
& H^{1}\left(M, S^{2}\right) \cap C^{0}\left(M, S^{2}\right)=\bigcup_{-\infty}^{+\infty} \varepsilon_{k}(M) \\
& =\bigcup_{-\infty}^{+\infty}\left\{u \in H^{1}\left(M, S^{2}\right) \cap C^{0}\left(M, S^{2}\right), \frac{1}{4 \pi} \int_{M} u \cdot\left(u_{x} \times u_{y}\right) d V o l_{M}=k\right\}
\end{aligned}
$$


where $Q(u)=\frac{1}{4 \pi} \int_{M} u \cdot\left(u_{x} \times u_{y}\right) d V o l_{M}$ is the degree and subscripts denote partial differentiation with respect to coordinates. We can also consider $S^{2}$ as $\mathbb{C} \cup\{\infty\}$ with the conformal metric $\frac{4}{\left(1+|z|^{2}\right)^{2}} d z d \bar{z}, u_{z}=\partial u, \partial=\frac{1}{2}\left(\frac{\partial}{\partial x}-i \frac{\partial}{\partial y}\right)$, and $u_{\bar{z}}=\bar{\partial} u$, $\bar{\partial}=\frac{1}{2}\left(\frac{\partial}{\partial x}+i \frac{\partial}{\partial y}\right)$. Then the energy functional is

$$
E(u)=\int_{M} \frac{4}{\left(1+|u|^{2}\right)^{2}}\left(\left|u_{z}\right|^{2}+\left|u_{\bar{z}}\right|^{2}\right) d z d \bar{z}
$$

and

$$
Q(u)=\frac{1}{\pi} \int_{M} \frac{1}{\left(1+|u|^{2}\right)^{2}}\left(\left|u_{z}\right|^{2}-\left|u_{\bar{z}}\right|^{2}\right) d z d \bar{z} .
$$

In the following, we will consider the energy functional on $\varepsilon_{1}(M)$. Our main result in this section is

THEOREM 2. We have

$$
\phi_{1}(M)=\inf _{v \in \varepsilon_{1}(M)} E(v)=4 \pi
$$

and $\phi_{1}(M)$ is attained if and only if $M$ is simply connected. Moreover, if $M$ is multiply connected and if $\left\{u_{n}\right\}_{n \in \mathbb{N}}$ is a minimizing sequence of $E$, then there exists $z_{0} \in M$ such that, modulo a subsequence,

$$
\frac{1}{2}\left|\nabla u_{n}\right|^{2} d V o l_{M} \longrightarrow 4 \pi \delta_{z_{0}}, \quad \text { in } \mathcal{R}(M),
$$

where $\delta_{z_{0}}$ is the Dirac mass concentrated at $z_{0} \in M$ and $\mathcal{R}(M)$ is the space of Radon measures on $M$ with finite masses.

In order to prove this result, we need the following lemmas.

LEMMA 1. (see [1] and [9]) Assume that $\varphi_{n}$ is a bounded sequence in $H^{1}(M, \mathbb{R}) \cap$ $L^{\infty}(M, \mathbb{R})$. Let $a_{n} \longrightarrow 0$ weakly in $H^{1}(M, \mathbb{R})$ and strongly in $L^{2}(M, \mathbb{R})$. Then for every $b \in H^{1}(M, \mathbb{R})$, we have

$$
\lim _{n \rightarrow \infty} \int_{M} \varphi_{n}\left(\left(a_{n}\right)_{x} b_{y}-\left(a_{n}\right)_{y} b_{x}\right) d V o l_{M}=0 .
$$

LEMMA 2. (Isoperimetric inequality see [22] and [9]) Assume that $\Psi=\left(\psi_{1}, \psi_{2}, \psi_{3}\right)$ $\in C^{0}\left(M, \mathbb{R}^{3}\right) \cap H^{1}\left(M, \mathbb{R}^{3}\right)$. Then,

$$
\left|\int_{M} \Psi \cdot\left(\Psi_{x} \times \Psi_{y}\right) d V o l_{M}\right|^{2} \leq \frac{1}{4 \pi}\left(\int_{M}\left|\Psi_{x} \times \Psi_{y}\right| d V o l_{M}\right)^{3} .
$$

Proof of Theorem 2. Obviously,

$$
E(u) \geq 4 \pi Q(u)=4 \pi, \quad \forall u \in \varepsilon_{1}(M)
$$


On the other hand, let $(U, z)$ be a coordinate neighborhood containing $p \in M$ such that $z(U)=B(0,1)=\{z \in \mathbb{C}:|z|<1\}$, we define for any $\epsilon>0$

$$
u_{p, \epsilon}(q)= \begin{cases}\frac{\epsilon}{z}, & \text { if }|z(q)| \leq \frac{1}{2} \\ \frac{\epsilon}{z}(3-4|z|), & \text { if } \frac{1}{2} \leq|z(q)| \leq \frac{3}{4} \\ 0, & \text { if } q \in M \backslash z^{-1}\left(B\left(0, \frac{3}{4}\right)\right) .\end{cases}
$$

It is clear that

$$
\lim _{\epsilon \rightarrow 0} E\left(u_{p, \epsilon}\right)=4 \pi \text {. }
$$

Therefore, we prove the first part of the Theorem. Using the Uniformization Theorem (see [12]), we may prove that $\phi_{1}(M)$ is achieved provided that $M$ is simply connected. Conversely, assume $u \in \varepsilon_{1}(M)$ such that $E(u)=4 \pi$. Then, $u$ satisfies equation (1.1) and is regular (see [10]). From (2.1) to (2.3), we deduce that $u_{\bar{z}}=0$; that is, $u$ is holomorphic. But, the degree of $u$ is one, and this is possible only if $M$ is simply connected. In particular we deduce that if $M$ is multiply connected, then Inequality (2.3) is strict.

Now let $\left\{u_{n}\right\}_{n \in \mathbb{N}}$ be a minimizing sequence of $E$ in $\varepsilon_{1}(M)$ for a Riemann surface $M$ of genus greater than 1 . Since $H^{1}$ is reflexive, we may assume that, modulo a subsequence, $u_{n} \longrightarrow u$ weakly in $H^{1}$ for some $u \in H^{1}$. But $H^{1}\left(M, S^{2}\right)$ is weakly closed, therefore $u \in H^{1}\left(M, S^{2}\right)$ and by weak lower semi-continuity, we have

$$
E(u) \leq \liminf _{n \rightarrow \infty} E\left(u_{n}\right)=4 \pi .
$$

Hence $u \in \varepsilon_{0}(M)$ because of (2.3). Set $\alpha_{n}=u_{n}-u$ so that (as in [9]) by lemma 1

$$
\begin{aligned}
1=Q\left(u_{n}\right) & =\frac{1}{4 \pi} \int_{M} u_{n} \cdot\left(\left(u_{n}\right)_{x} \times\left(u_{n}\right)_{y}\right) d V o l_{M} \\
& =\frac{1}{4 \pi} \int_{M} u_{n} \cdot\left(\left(\alpha_{n}\right)_{x} \times\left(\alpha_{n}\right)_{y}\right) d V o l_{M}+\frac{1}{4 \pi} \int_{M} u \cdot\left(u_{x} \times u_{y}\right) d V o l_{M}+o(1) \\
& =\frac{1}{4 \pi} \int_{M} u_{n} \cdot\left(\left(\alpha_{n}\right)_{x} \times\left(\alpha_{n}\right)_{y}\right) d V o l_{M}+o(1)
\end{aligned}
$$

Therefore,

$$
E\left(\alpha_{n}\right)=\frac{1}{2} \int_{M}\left|\nabla \alpha_{n}\right|^{2} d V o l_{M} \geq\left|\int_{M} u_{n} \cdot\left(\left(\alpha_{n}\right)_{x} \times\left(\alpha_{n}\right)_{y}\right) d V o l_{M}\right| \geq 4 \pi+o(1) .
$$

On the other hand, we have

$$
4 \pi+o(1)=E\left(u_{n}\right)=E(u)+E\left(\alpha_{n}\right)+o(1),
$$

which implies

$$
E(u)=0 \quad \text { and } \quad E\left(\alpha_{n}\right)=4 \pi+o(1)
$$

Hence, $u$ is constant. Set $\mu_{n}=\frac{1}{2}\left|\nabla \alpha_{n}\right|^{2} d V o l_{M}$ and $\nu_{n}=\alpha_{n} \cdot\left(\left(\alpha_{n}\right)_{x} \times\left(\alpha_{n}\right)_{y}\right) d V o l_{M}$. Clearly, $\mu_{n}(M)=4 \pi+o(1), \nu_{n}(M)=4 \pi$ and $\left|\nu_{n}\right| \ll \mu_{n}$. Therefore $\left\{\mu_{n}\right\}$ and $\left\{\nu_{n}\right\}$ 
are bounded in $\mathcal{R}(M)$. Modulo a subsequence, we may assume that $\mu_{n} \rightarrow \mu, \nu_{n} \rightarrow \nu$ weakly in the sense of measures where $\mu$ and $\nu$ are bounded measures on $M$. Moreover, $\mu(M)=4 \pi$ and $\nu(M)=4 \pi$ since $M$ is compact. Choose $\xi \in C^{\infty}(M, \mathbb{R})$. Applying lemma 2 , we have

$$
\left|\int_{M} \xi \alpha_{n} \cdot\left(\left(\xi \alpha_{n}\right)_{x} \times\left(\xi \alpha_{n}\right)_{y}\right) d V o l_{M}\right|^{2} \leq \frac{1}{32 \pi}\left(\int_{M}\left|\nabla\left(\xi \alpha_{n}\right)\right|^{2} d V o l_{M}\right)^{3} .
$$

It is clear that

$$
\begin{aligned}
\int_{M} \xi \alpha_{n} \cdot\left(\left(\xi \alpha_{n}\right)_{x} \times\left(\xi \alpha_{n}\right)_{y}\right) d V o l_{M} & =\int_{M} \xi^{3} \alpha_{n} \cdot\left(\left(\alpha_{n}\right)_{x} \times\left(\alpha_{n}\right)_{y}\right) d V o l_{M}+o(1) \\
\int_{M}\left|\nabla\left(\xi \alpha_{n}\right)\right|^{2} d V o l_{M} & =\int_{M} \xi^{2}\left|\nabla \alpha_{n}\right|^{2} d V o l_{M}+o(1)
\end{aligned}
$$

since $\alpha_{n} \longrightarrow 0$ strongly in $L^{2}$ and $\left\|\alpha_{n}\right\|_{L^{\infty}} \leq 2$. Consequently, we get

$$
\left|\int_{M} \xi^{3} \alpha_{n} \cdot\left(\left(\alpha_{n}\right)_{x} \times\left(\alpha_{n}\right)_{y}\right) d V o l_{M}\right|^{2} \leq \frac{1}{32 \pi}\left(\int_{M} \xi^{2}\left|\nabla \alpha_{n}\right|^{2} d V o l_{M}+o(1)\right)^{3} .
$$

Passing to the limit as $n \rightarrow \infty$, there holds

$$
\left|\int_{M} \xi^{3} d \nu\right|^{2} \leq \frac{1}{4 \pi}\left(\int_{M} \xi^{2} d \mu\right)^{3}, \quad \forall \xi \in C^{\infty}(M, \mathbb{R})
$$

By approximation, therefore,

$$
|\nu(E)|^{\frac{2}{3}} \leq\left(\frac{1}{4 \pi}\right)^{\frac{1}{3}} \mu(E) \quad(E \subset M, E \text { borel })
$$

which implies

$|\nu(A)|^{\frac{2}{3}} \leq\left(\frac{1}{4 \pi}\right)^{\frac{1}{3}} \mu(A)$ and $|\nu(M \backslash A)|^{\frac{2}{3}} \leq\left(\frac{1}{4 \pi}\right)^{\frac{1}{3}} \mu(M \backslash A)$ for any Borel set $A$.

However, $\mu(M)=\nu(M)=4 \pi$. Thus, we deduce that

$$
\nu(A)=\mu(A)=0 \quad \text { or } \quad \nu(M \backslash A)=\mu(M \backslash A)=0 .
$$

Since it is true for all $A$, that implies the assertion of Theorem 2.

Now let $\tilde{g}$ be a metric compatible with the complex structure on $M$. By NashMoser's Theorem, $(M, \tilde{g})$ can be isometrically embedded in some Euclidean space $\mathbb{R}^{k}$ and we denote this embedding by $I$. We analyze the topology of the energy level sets $E_{M}^{4 \pi+\gamma}=\left\{u \in \varepsilon_{1}(M): E(u) \leq \gamma+4 \pi\right\}$ for small $\gamma>0$. For this purpose, we introduce a map $C$ from $\varepsilon_{1}(M)$ into $\mathbb{R}^{k}$

$$
\begin{aligned}
C: \varepsilon_{1}(M) & \longrightarrow \mathbb{R}^{k} \\
u & \longmapsto \frac{1}{8 \pi} \int_{M} I(q)|\nabla u|^{2}(q) d V \operatorname{Vol}_{M} .
\end{aligned}
$$

There is a neighborhood $U_{\delta}=\left\{x \in \mathbb{R}^{k}: \operatorname{dist}(x, M) \leq \delta\right\}$ of $M$ in $\mathbb{R}^{k}$ on which the nearest projection $P$ from $U_{\delta}$ onto $M$ is continuous. According to Theorem 2, we can construct a map $\bar{\pi}$ for all small $\gamma>0$

$$
\begin{array}{lll}
\bar{\pi}: & E_{M}^{4 \pi+\gamma} & \longrightarrow \\
u & \longmapsto & M \\
& &
\end{array}
$$


Note that, the construction given in (2.4) shows that $\bar{\pi}$ is surjective. For some $\gamma$ fixed, we choose a suitable $\epsilon_{0}$ such that for any $p \in M$, we have $u_{p, \epsilon_{0}} \in E_{M}^{4 \pi+\gamma}$. So we define another continuous map $\tau$ from $M$ to $E_{M}^{4 \pi+\gamma}$

$$
\tau(p)=u_{p, \epsilon_{0}} .
$$

Using Theorem 2, we deduce that $\tau \circ \bar{\pi}$ and $I d_{E_{M}^{4 \pi+\gamma}}$ are homotopic and that $\bar{\pi} \circ \tau$ and $I d_{M}$ are so if $\gamma$ is sufficiently small; i.e., $E_{M}^{4 \pi+\gamma}$ and $M$ are of the same homotopy type.

3. Study of nonconstant harmonic maps. In this section, we will analyze the energy of nonconstant harmonic maps from closed Riemann surface of genus $g$ to $S^{2}$. For this purpose, we consider Schiffer's interior variation in $T_{g}$. Assume distinct points $r_{1}, \ldots, r_{3 g-3}$, are given on $R_{1}$. We take coordinate neighborhoods $\left(U_{j}, z_{j}^{*}\right)$ of every $r_{i}$ so that

$$
\begin{aligned}
& z_{j}^{*}\left(r_{j}\right)=0 \\
& z_{j}^{*}\left(U_{j}\right)=\{z \in \mathbb{C}:|z|<2\}, \quad j=1, \ldots, 3 g-3 ; \\
& \overline{U_{j}} \cap \overline{U_{k}}=\emptyset, \quad j \neq k ; \\
& \overline{U_{j}} \cap \overline{V_{1}}=\emptyset,
\end{aligned}
$$

where $V_{1}$ is defined in $\S 1$. Set $D_{j}=\left(z_{j}^{*}\right)^{-1}(\{z \in \mathbb{C}:|z|<1\})$. For any complex number $\epsilon_{j}^{\prime}$ with $\left|\epsilon_{j}^{\prime}\right|<\frac{1}{2}$, consider the mapping

$$
z_{j, \epsilon_{j}^{\prime}}^{*}(p)=z_{j}^{*}(p)+\frac{\epsilon_{j}^{\prime}}{z_{j}^{*}(p)}, \quad \forall p \in D_{j} .
$$

When $\left|\epsilon_{j}^{\prime}\right|$ is sufficiently small, $z_{j, \epsilon_{j}^{\prime}}^{*}\left(\partial D_{j}\right)$ is a simple closed curve in the $z_{j, \epsilon_{j}^{\prime}}^{*}$-plane, which is denoted by $C_{j, \epsilon_{j}^{\prime}}$, and $z_{j, \epsilon_{j}^{\prime}}^{*}$ gives a conformal mapping of a suitable neighborhood $A_{j, \epsilon_{j}^{\prime}}$ of $\partial D_{j}$. Now exclude $D_{j}$ from $R_{1}$ and paste the domain $D_{j, \epsilon_{j}^{\prime}}$ in the $z_{j, \epsilon_{j}^{\prime}}^{*}$-plane surrounded by $C_{j, \epsilon_{j}^{\prime}}$. Thus, for $\epsilon_{1}$ and $\epsilon_{2}$ fixed, we can construct a family $\left\{R_{\epsilon_{1}, \epsilon_{2}, \epsilon^{\prime}}\right\}$ of Riemann surfaces depending on complex parameters $\epsilon^{\prime}=\left(\epsilon_{1}^{\prime}, \ldots, \epsilon_{3 g-3}^{\prime}\right)$ (see [12]).

We also need the Hodge star operator. For each $\alpha \in \wedge^{p}(M)$, we associate to $\alpha$ a $(2-p)$-form $* \alpha$, called the adjoint of $\alpha$, defined as follows:

$$
* 1=\eta, * d x=d y, * d y=-d x, * \eta=1,
$$

where $\eta$ is the oriented volume element on $M$. And we define $\delta \alpha$ by

$$
\delta \alpha=(-1)^{p} *^{-1} d * \alpha, \quad \text { where } p=\operatorname{deg}(\alpha) .
$$

Then, the Laplace operator $\Delta$ is defined by

$$
\Delta=d \delta+\delta d
$$

THEOREM 3. Under the above assumptions, there exists $\beta>0$ such that for any Riemann surface $R_{\epsilon_{1}, \epsilon_{2}, \epsilon^{\prime}}$ with $\left|\epsilon_{i}\right|<1$ and $\left|\epsilon_{j}^{\prime}\right|<\frac{1}{2}$ and a nonconstant harmonic map $u$ from $R_{\epsilon_{1}, \epsilon_{2}, \epsilon^{\prime}}$ to $S^{2}$, we have

$$
E(u) \geq \beta
$$


As a direct consequence of Schiffer's interior variation, we obtain the following corollary

CoROLlaRY. The energy of nonconstant harmonic maps to $S^{2}$ is uniformly bounded from below on a open set of $T_{g}$.

REMARK. $\mathcal{M}_{g}$ is identified with the quotient space $T_{g} / \operatorname{Mod}_{g}$, where $\operatorname{Mod}_{g}$ is the Teichmüller modular group. Hence, our result is also true on a open set of $\mathcal{M}_{g}$ equipped with the quotient topology.

In order to prove the theorem, we need a technical lemma.

Lemma 3. (see [10] and [11]) Let $u: B(0,1)=\{z \in \mathbb{C}:|z|<1\} \longrightarrow S^{2}$ be a harmonic map. Then there exists a positive constant $C$ such that for any $z_{1}$, $z_{2} \in B\left(0, \frac{1}{2}\right)$,

$$
\left|u\left(z_{1}\right)-u\left(z_{2}\right)\right| \leq C\left(\int_{B}|\nabla u|^{2} d z d \bar{z}\right)^{\frac{1}{2}}\left(\left(\int_{B}|\nabla u|^{2} d z d \bar{z}\right)^{\frac{1}{2}}+1\right) .
$$

Proof. In view of (1.1), there exists $G$ from $B$ to $\mathbb{R}^{3}$ satisfying

$$
\frac{\partial G}{\partial x}=u \times \frac{\partial u}{\partial y}, \quad \frac{\partial G}{\partial y}=-u \times \frac{\partial u}{\partial x} .
$$

By the Courant-Lebesgue Lemma, there exists some $r \in\left(\frac{1}{2}, 1\right)$ for which $\left.u\right|_{\partial B(0, r)}$ is absolutely continuous and

$$
\left|u\left(z_{3}\right)-u\left(z_{4}\right)\right| \leq\left(\frac{8 \pi}{\log 2}\right)^{\frac{1}{2}}\left(\int_{B}|\nabla u|^{2} d z d \bar{z}\right)^{\frac{1}{2}}
$$

for all $z_{3}, z_{4} \in \partial B(0, r)$. In $B(0, r)$, we will decompose $u$ into its harmonic $\left(u_{0}\right)$ and non harmonic $\left(u_{1}\right)$ components

$$
u=u_{0}+u_{1}
$$

where

$$
\begin{gathered}
\left\{\begin{aligned}
\Delta u_{0}=0 & \text { in } B(0, r) \\
u_{0}=u & \text { on } \partial B(0, r),
\end{aligned}\right. \\
\left\{\begin{aligned}
\Delta u_{1}=\Delta u=u_{x} \times G_{y}+G_{x} \times u_{y} & \text { in } B(0, r) \\
u_{1}=0 & \text { on } \partial B(0, r) .
\end{aligned}\right.
\end{gathered}
$$

Thanks to the Wente's inequality (see [1] and [22]), we obtain

$$
\left\|u_{1}\right\|_{C^{0}} \leq C\|\nabla u\|_{L^{2}}\|\nabla G\|_{L^{2}} .
$$

And by the Maximum Principle, we deduce that for all $z_{1}, z_{2} \in B(0, r)$

$$
\left|u_{0}\left(z_{1}\right)-u_{0}\left(z_{2}\right)\right| \leq \sup _{z_{3}, z_{4} \in \partial B(0, r)}\left|u_{0}\left(z_{3}\right)-u_{0}\left(z_{4}\right)\right|
$$


Combining (3.5)-(3.9), we establish (3.4).

Proof of Theorem 3. Let us decompose $R_{\epsilon_{1}, \epsilon_{2}, \epsilon^{\prime}}=F \cup\left(R_{\epsilon_{1}, \epsilon_{2}, \epsilon^{\prime}} \backslash F\right)$, where $F=\left\{z \in R_{\epsilon_{1}, \epsilon_{2}, \epsilon^{\prime}}: \operatorname{rank}(\nabla u)(z) \leq 1\right\}$ is the set of degenerated points. Denote by $\mathcal{H}^{2}$ the 2-dimensional Hausdorff measure in $\mathbb{R}^{3}$. Applying Sard's Lemma,

$$
\mathcal{H}^{2}(u(F))=0 .
$$

For each $z \in R_{\epsilon_{1}, \epsilon_{2}, \epsilon^{\prime}} \backslash F$, there exists a closed neighborhood $G_{z}$ of $z$ such that $\left.u\right|_{G_{z}}: G_{z} \longrightarrow \overline{B\left(u(z), r_{z}\right)}$ is a diffeomorphism. Notice that $u\left(R_{\epsilon_{1}, \epsilon_{2}, \epsilon^{\prime}} \backslash F\right)$ is open. Then, using Vitali's covering theorem, we can choose a countable family of disjoint $\operatorname{discs} \overline{B\left(u\left(z_{i}\right), r_{z_{i}}\right)}$ satisfying

$$
u\left(R_{\epsilon_{1}, \epsilon_{2}, \epsilon^{\prime}} \backslash F\right) \subset \bigcup_{i=1}^{\infty} B\left(u\left(z_{i}\right), 5 r_{z_{i}}\right) .
$$

Consequently, there exists a positive constant $C$ such that

$$
\begin{aligned}
\mathcal{H}^{2}\left(u\left(R_{\epsilon_{1}, \epsilon_{2}, \epsilon^{\prime}} \backslash F\right)\right) & \leq \mathcal{H}^{2}\left(\bigcup_{i=1}^{\infty} B\left(u\left(z_{i}\right), 5 r_{z_{i}}\right)\right) \\
& \leq \sum_{i=1}^{\infty} \mathcal{H}^{2}\left(B\left(u\left(z_{i}\right), 5 r_{z_{i}}\right)\right) \\
& \leq C \sum_{i=1}^{\infty} \mathcal{H}^{2}\left(B\left(u\left(z_{i}\right), r_{z_{i}}\right)\right) \\
& \leq C \sum_{i=1}^{\infty} \int_{G_{z_{i}}}\left|u \cdot\left(u_{x} \times u_{y}\right)\right| d z d \bar{z} \\
& \leq C E(u) .
\end{aligned}
$$

On the other hand, according to Lemma 3 , for some $s_{1}, s_{2} \in S^{2}$ and for any sufficiently small $E(u)$, we have

$$
u\left(R_{1} \backslash\left(V_{1} \cup\left(\bigcup_{j=1}^{3 g-3} U_{j}\right)\right)\right) \subset B\left(s_{1}, C \sqrt{E(u)}\right)
$$

and

$$
u\left(R_{2} \backslash V_{2}\right) \subset B\left(s_{2}, C \sqrt{E(u)}\right)
$$

Let $Q$ be the antipodal mapping on $S^{2}$; that is, $Q(v)=-v$ for all $v \in S^{2}$. Combining equations (3.10)-(3.14), we have

$$
\mathcal{H}^{2}(K \cup Q(K)) \leq C E(u), \quad \text { for sufficiently small } E(u),
$$

where $K=u\left(R_{\epsilon_{1}, \epsilon_{2}, \epsilon^{\prime}}\right) \cup \overline{B\left(s_{1}, C \sqrt{E(u)}\right)} \cup \overline{B\left(s_{2}, C \sqrt{E(u)}\right)}$. However, $K \cup Q(K)$ is closed since $u\left(R_{\epsilon_{1}, \epsilon_{2}, \epsilon^{\prime}}\right)$ is compact. Thus, choosing $E(u)$ sufficiently small, we can find a pair of antipodal points $v_{1}$ and $-v_{1}$ on $S^{2}$ such that $v_{1} \notin K \cup Q(K)$ and $-v_{1} \notin K \cup Q(K)$. Without loss of generality, we suppose that $v_{1}=(0,0,1)$ is the north pole. Denote $K_{1}=S^{2} \backslash\left\{v_{1},-v_{1}\right\}$ and let

$$
u_{\sharp}: \pi_{1}\left(R_{\epsilon_{1}, \epsilon_{2}, \epsilon^{\prime}}\right) \longrightarrow \pi_{1}\left(K_{1}\right)=\mathbb{Z}
$$


be the induced map on the fundamental groups. We claim that if $E(u) \leq \beta$ (here $\beta$ is a uniform constant), then $u_{\sharp}$ is trivial. Indeed, if $E(u)$ is sufficiently small, we can choose a simply connected subset $K_{2}$ of $K_{1}$ containing $\overline{B\left(s_{i}, C \sqrt{E(u)}\right)}$, for $i=1,2$, and a deformation map of $K_{1}$ such that

$$
\begin{aligned}
& f: K_{1} \times[0,1] \longrightarrow K_{1}, \\
& f(0, \cdot)=I d, \\
& f\left(K_{2}, t\right) \subset K_{2}, \quad \text { for all } t \in[0,1], \\
& f\left(K_{2}, 1\right)=\left\{s_{1}\right\} .
\end{aligned}
$$

Denote $f_{t}=f(t, \cdot)$. Hence, $u_{\sharp}=\left(f_{1} \circ u\right)_{\sharp}$. We can write $f_{1} \circ u=\check{f} \circ P_{1}$, where

$$
\begin{aligned}
P_{1}: R_{\epsilon_{1}, \epsilon_{2}, \epsilon^{\prime}} & \longrightarrow K_{3}=R_{\epsilon_{1}, \epsilon_{2}, \epsilon^{\prime}} /\left(\left(R_{1} \backslash\left(V_{1} \cup \bigcup_{i=1}^{3 g-3} U_{i}\right)\right) \cup\left(R_{2} \backslash V_{2}\right)\right) \\
z & \longrightarrow[z]
\end{aligned}
$$

is a projection from $R_{\epsilon_{1}, \epsilon_{2}, \epsilon^{\prime}}$ onto $K_{3}$ which is equipped with quotient topology, and $\check{f}$ is a continuous mapping from $K_{3}$ to $K_{1}$ since $f_{1}\left(K_{2}\right)=\left\{s_{1}\right\}$. Notice that $K_{3}$ is simply connected, the claim is proved.

Denote $u=\left(u^{1}, u^{2}, u^{3}\right)$. Set $u^{1}+i u^{2}=\sqrt{\left(u^{1}\right)^{2}+\left(u^{2}\right)^{2}} e^{i \psi}$. In fact, $\psi$ can be defined locally or on a simply connected subset of $R_{\epsilon_{1}, \epsilon_{2}, \epsilon^{\prime}}$. But $u_{\sharp}\left(\pi_{1}\left(R_{\epsilon_{1}, \epsilon_{2}, \epsilon^{\prime}}\right)\right)$ is trivial in $\pi_{1}\left(K_{1}\right)$. So $\psi$ is defined on the whole $R_{\epsilon_{1}, \epsilon_{2}, \epsilon^{\prime}}$. It follows from (1.1) that

$$
\operatorname{div}\left(\left(\left(u^{1}\right)^{2}+\left(u^{2}\right)^{2}\right) \nabla \psi\right)=\operatorname{div}\left(u^{1} \nabla u^{2}-u^{2} \nabla u^{1}\right)=0
$$

or equivalently, using the language of differential forms,

$$
\delta\left(\left(\left(u^{1}\right)^{2}+\left(u^{2}\right)^{2}\right) d \psi\right)=0
$$

Consequently,

$$
0=\int_{R_{\epsilon_{1}, \epsilon_{2}, \epsilon^{\prime}}} \delta\left(\left(\left(u^{1}\right)^{2}+\left(u^{2}\right)^{2}\right) d \psi\right) \psi d z d \bar{z}=\int_{R_{\epsilon_{1}, \epsilon_{2}, \epsilon^{\prime}}}\left(\left(u^{1}\right)^{2}+\left(u^{2}\right)^{2}\right)|d \psi|^{2} d z d \bar{z}
$$

which implies that $\psi$ is a constant mapping. That is, $u\left(R_{\epsilon_{1}, \epsilon_{2}, \epsilon^{\prime}}\right)$ is contained in a great circle. Replacing $\left\{v_{1},-v_{1}\right\}$ by another pair of antipodal points $\left\{v_{1}^{\prime},-v_{1}^{\prime}\right\}$ and proceeding similarly, we deduce that $u$ is a constant mapping. This completes the proof.

4. Proof of Theorem 1. First, we recall some facts which can be found in [13] (§4.2), primarily due to Sack-Uhlenbeck [17].

LEMMA 4. Let $M$ be a compact Riemann surface without boundary, and $N$ be a compact Riemannian manifold without boundary. Let $A$ be a compact parameter space and let $h_{0}: M \times A \longrightarrow N$ be continuous. Let $H$ be the class of all maps homotopic to $h_{0}$ and put

$$
\kappa=\inf _{h \in H} \sup _{t \in A} E(h(\cdot, t)) .
$$

In case $\partial A \neq \emptyset,\left.h\right|_{M \times \partial A}$ is fixed in such a way that the above supremum cannot be attained on $\partial A$. Then there exists a harmonic map

$$
u_{0}: M \longrightarrow N
$$


and possibly also some nontrivial conformal harmonic maps

$$
u_{i}: S^{2} \longrightarrow N \quad(i=1, \ldots, m)
$$

with

$$
E\left(u_{0}\right)+\sum_{i=1}^{m} E\left(u_{i}\right)=\kappa .
$$

Here $\left(u_{0} ; u_{1}, \ldots, u_{m}\right)$ represents a saddle point corresponding to $H$ in the sense that there exist sequences $\left\{h_{n}\right\} \subset H,\left\{t_{n}\right\} \subset A$ and points $x_{1}, . ., x_{k} \in M, k \leq m$ (if $m \geq 1$ ) with

$$
\begin{array}{ll}
E\left(h_{n}\left(\cdot, t_{n}\right)\right) \longrightarrow \kappa & ; \\
h_{n}\left(\cdot, t_{n}\right) \longrightarrow u_{0} & \text { weakly in } H^{1} ; \\
h_{n}\left(\cdot, t_{n}\right) \longrightarrow u_{0} & \text { uniformly on each compact subset of } M \backslash\left\{x_{1}, . ., x_{k}\right\} .
\end{array}
$$

Furthermore, for each $i \in\{1, \ldots, m\}$, there exists a sequence $\left\{\lambda_{n}^{i}\right\}_{n \in \mathbb{N}} \subset \mathbb{R}^{+}, \lambda_{n}^{i} \rightarrow 0$ as $n \rightarrow \infty$ with,

$$
h_{n}\left(\left(\frac{\rho}{\lambda_{n}^{i}}, \varphi\right), t_{n}\right) \longrightarrow u_{i},
$$

where $(\rho, \varphi)$ are polar coordinates centered at some $x_{j, n}$ with $x_{j, n} \rightarrow x_{j}(1 \leq j \leq k)$.

LEMMA 5. Let $u$ be a harmonic map from $S^{2}$ to $S^{2}$. Then $u$ is conformal and

$$
E(u)=4 \pi \operatorname{deg}(u)
$$

In view of the results in $\S 2$, we know that the energy level sets near the minimum have a nontrivial topology. Thus, we can construct a nontrivial loop in such level sets. However, it will be contractible in higher energy level sets. With the help of the above lemmas, we obtain a critical value of $E$ using the Minimax Principle for the energy $E$ based on this loop. In case no blow-up can occur, we prove the assertion.

For simplicity, we use conformal coordinates $z \in \mathbb{C} \cup\{\infty\}$ on $S^{2}$, such that $q_{1}=0$ and $q_{2}=\infty$. Choose a function $\psi(z) \in C_{0}^{\infty}\left(\mathbb{R}^{2}, \mathbb{R}\right)$ such that $\operatorname{supp}(\psi) \subset B(0,1)$, $0 \leq \psi \leq 1$ and $\psi(z)=1$ if $|z| \leq \frac{1}{2}$. We write $S^{2}=S_{+}^{2} \cup S_{-}^{2}$ where $S_{+}^{2}=\{v=$ $\left.\left(v^{1}, v^{2}, v^{3}\right) \in S^{2}: v^{3} \geq 0\right\} \simeq \overline{B(0,1)}$ and $S_{-}^{2}=\left\{v=\left(v^{1}, v^{2}, v^{3}\right) \in S^{2}: v^{3} \leq 0\right\} \simeq$ $\mathbb{C} \cup\{\infty\} \backslash B(0,1)$. Similarly, we write $R_{\epsilon_{1}, \epsilon_{2}, \epsilon^{\prime}}=R_{\epsilon_{1}, \epsilon_{2}, \epsilon^{\prime}}^{+} \cup R_{\epsilon_{1}, \epsilon_{2}, \epsilon^{\prime}}^{-}$where $R_{\epsilon_{1}, \epsilon_{2}, \epsilon^{\prime}}^{+}$ (resp. $R_{\epsilon_{1}, \epsilon_{2}, \epsilon^{\prime}}^{-}$) is obtained by pasting $R_{1}$ (resp. $R_{2}$ ) to $S_{+}^{2}$ (resp. $S_{-}^{2}$ ). For $z^{*} \in S^{1}$ and $0 \leq t<1$, we set $\sigma_{z^{*}, t}(z)=\frac{z+t z^{*}}{1+t \overline{z^{*}} z}$. We construct a continuous map $h$ from $B(0,1) \times R_{\epsilon_{1}, \epsilon_{2}, \epsilon^{\prime}}$ to $S^{2}$ by

$$
h\left(z^{*}, t, z\right)= \begin{cases}t z^{*}, & \text { if } z \in R_{\epsilon_{1}, \epsilon_{2}, \epsilon^{\prime}}^{+} \backslash U_{1, \epsilon_{1}}^{\prime} \\ \left(\sigma_{z^{*}, t}(z)-t z^{*}\right)\left(1-\psi\left(\frac{z}{2\left|\epsilon_{1}\right|}\right)\right)+t z^{*}, & \text { if }\left|\epsilon_{1}\right| \leq|z| \leq 2\left|\epsilon_{1}\right| \\ \sigma_{z^{*}, t}(z), & \text { if } 2\left|\epsilon_{1}\right| \leq|z| \leq \frac{1}{2\left|\epsilon_{2}\right|} \\ \left(\sigma_{z^{*}, t}(z)-\frac{1}{t \overline{z^{*}}}\right)\left(1-\psi\left(\frac{1}{2\left|\epsilon_{2}\right| z}\right)\right)+\frac{1}{\overline{t z^{*}}}, & \text { if } \frac{1}{2\left|\epsilon_{2}\right|} \leq|z| \leq \frac{1}{\left|\epsilon_{2}\right|} \\ \frac{1}{t \overline{z^{*}}}, & \text { if } z \in R_{\epsilon_{1}, \epsilon_{2}, \epsilon^{\prime}}^{-} \backslash U_{2, \epsilon_{2}}^{\prime}\end{cases}
$$


Notice that, for any $z^{*}, t$, the map $z \longmapsto h\left(z^{*}, t, z\right)$ is constant on the glued surfaces $R_{1}$ and $R_{2}$; if $t \longrightarrow 1$, it concentrates at some point on the equator of $S^{2}$, parametrized by $z^{*}$. A direct computation shows

$$
\lim _{t \rightarrow 1} E\left(h\left(z^{*}, t, \cdot\right)\right)=4 \pi \quad \text { uniformly on } S^{1}
$$

and

$$
\lim _{\epsilon_{1}, \epsilon_{2} \rightarrow 0} \sup _{z^{*}} E\left(h\left(z^{*}, t, \cdot\right)\right)=4 \pi .
$$

Thus, there is $\alpha>0$ such that

$$
\sup _{B(0,1)} E\left(h\left(z^{*}, t, \cdot\right)\right)<4 \pi+\beta<8 \pi \quad \text { for any }\left|\epsilon_{i}\right|<\alpha \text { and }\left|\epsilon_{j}^{\prime}\right|<\frac{1}{2} .
$$

Now, fix $\epsilon_{i}$ and $\epsilon^{\prime}$. We choose some small $\mu_{0}$ in such a way that $\bar{\pi}$ in $\S 2$ can be defined continuously from $E_{R_{\epsilon_{1}, \epsilon_{2}, \epsilon^{\prime}}^{4 \pi+\mu_{0}}}$ to $R_{\epsilon_{1}, \epsilon_{2}, \epsilon^{\prime}}$. By (4.3) and Theorem 2, we can find $0<t_{0}<1$ such that $E\left(h\left(z^{*}, t_{0}, \cdot\right)\right)<4 \pi+\mu_{0}$ for all $z^{*} \in S^{1}$ and $z^{*} \rightarrow \bar{\pi}\left(h\left(z^{*}, t_{0}, \cdot\right)\right)$ is a nontrivial loop in $\pi_{1}\left(R_{\epsilon_{1}, \epsilon_{2}, \epsilon^{\prime}}\right)$ since $\lim _{t \rightarrow 1} \bar{\pi}\left(h\left(z^{*}, t, \cdot\right)\right)=z^{*} \in R_{\epsilon_{1}, \epsilon_{2}, \epsilon^{\prime}}$. We consider $h$ as a function defined on $B\left(0, t_{0}\right) \times R_{\epsilon_{1}, \epsilon_{2}, \epsilon^{\prime}}$ and let $H$ be the homotopy classes of $h$. Set

$$
\kappa_{\epsilon_{1}, \epsilon_{2}, \epsilon^{\prime}}=\inf _{\bar{f} \in H} \sup _{z^{*} t \in B\left(0, t_{0}\right)} E\left(\bar{f}\left(z^{*}, t, \cdot\right)\right)
$$

LEMMA 6. We have

$$
\kappa_{\epsilon_{1}, \epsilon_{2}, \epsilon^{\prime}} \geq 4 \pi+\mu_{0}
$$

Proof. We suppose that $\kappa_{\epsilon_{1}, \epsilon_{2}, \epsilon^{\prime}}<4 \pi+\mu_{0}$. Then for some $\bar{f} \in H$, we have

$$
E\left(\bar{f}\left(z^{*}, t, \cdot\right)\right)<4 \pi+\mu_{0}, \quad \text { for all } t \in\left[0, t_{0}\right] \text { and } z^{*} \in S^{1} .
$$

Hence, we can construct a deformation map $\tilde{f}$ from $B\left(0, t_{0}\right)$ to $R_{\epsilon_{1}, \epsilon_{2}, \epsilon^{\prime}}$ by

$$
\begin{aligned}
\tilde{f}: & B\left(0, t_{0}\right) \\
\left(z^{*}, t\right) & \longrightarrow R_{\epsilon_{1}, \epsilon_{2}, \epsilon^{\prime}}
\end{aligned}
$$

that is, $\tilde{f}$ is a contraction of $S^{1}$ in $R_{\epsilon_{1}, \epsilon_{2}, \epsilon^{\prime}}$. This contradiction completes our proof.

Proof of Theorem 1'. Obviously, $\kappa_{\epsilon_{1}, \epsilon_{2}, \epsilon^{\prime}}<4 \pi+\beta$, where $\beta$ is chosen as in Theorem 3. Now, according to Lemma 4 and Lemma 5 , there exists a harmonic map

$$
u_{0}: R_{\epsilon_{1}, \epsilon_{2}, \epsilon^{\prime}} \longrightarrow S^{2}
$$

and possibly also some non-trivial conformal harmonic maps

$$
u_{i}: S^{2} \longrightarrow S^{2}, \quad(i=1, \ldots, m)
$$

with

$$
\kappa_{\epsilon_{1}, \epsilon_{2}, \epsilon^{\prime}}=E\left(u_{0}\right)+\sum_{i=1}^{m} E\left(u_{i}\right)=4 k \pi+E\left(u_{0}\right), \quad \text { for some } k \in \mathbb{N}
$$


Finally, notice that $4 \pi+\mu_{0} \leq \kappa_{\epsilon_{1}, \epsilon_{2}, \epsilon^{\prime}}<4 \pi+\beta<8 \pi$. So $k=0$ or $k=1$. But, in view of Theorem 3 , the latter implies that $u_{0}$ is a constant map, which contradicts $\kappa_{\epsilon_{1}, \epsilon_{2}, \epsilon^{\prime}} \geq 4 \pi+\mu_{0}$. Thus, $u_{0}$ is harmonic map of degree 1 from $R_{\epsilon_{1}, \epsilon_{2}, \epsilon^{\prime}}$ to $S^{2}$.

REMARK. In general, if we attach several small handles to the unit sphere $S^{2}$ as in $\S 1$, we have the same result.

Acknowlegements. The author would like to thank Professor J.M. Coron for suggesting him to realize that work and stimulating discussions. He is also grateful to Professor F. Hélein for his helpful discussions and suggestions and thanks the referees for their comments.

\section{REFERENCES}

[1] H. Brezis AND J. M. CoRon, Multiple solutions of H-systemes and Rellich's conjecture, Comm. Pure. Appl. Math, 37 (1984), pp. 149-187.

[2] H. BREzIS AND J. M. CoRon, Convergence of solutions of $H$-systemes or how to blow bubbles, Arch. Rat. Mech. Anal, 89 (1985), pp. 21-56.

[3] K. C. CHANG, Heat flow and boundary value problem for harmonic maps, Ann. I. H. P., Analyse non-linéaire, 6 (1989), pp. 363-395.

[4] J. M. Coron, Topologie et cas limite des injections de Sobolev, C. R. Acad. Sc. Paris, Ser. I, 299 (1984), pp. 209-212.

[5] J. M. CORON, private communication.

[6] R. Courant, Dirichlet's Principle, Conformal Mapping, and Minimal Surfaces, Interscience, New York, 1975.

[7] J. Eells ANd L. Lemaire, A report on harmonic maps, Bull. London. Math. Soc., 10 (1978), pp. 1-108.

[8] J. Eells AND J. C. Wood, Restrictions on harmonic maps of surfaces, Topology, 299 (1975), pp. 263-266.

[9] Y. GE, Estimations of the best constant involving the $L^{2}$ norm in Wente's inequality and compact $H$-surfaces into Euclidean space, Part I and Part II, COCV, 3 (1998), pp. 263300.

[10] F. HÉLEIN, Régularité des applications faiblement harmoniques entre une surface et une sphère, C. R. Acad. Sci. Paris, 311 (1990), pp. 519-524.

[11] F. HÉlein, Applications Harmoniques, Lois de Conservation et Repère Mobile, Diderot éditeur, Paris, New York, Amsterdam, 1996.

[12] Y. Imayoshi and M. TANiguchi, An Introduction to Teichmüller Spaces, Springer, Tokyo, 1992.

[13] J. Jost, Two-Dimensional Geometric Variational Problems, Wiley, 1991.

[14] L. Lemaire, Applications harnomiques de surfaces riemanniennes, J. Diff. Geom., 13 (1978), pp. 51-78.

[15] P. L. LIONS, The concentration-compactness principle in the calculus of variations: The limit case. Part I and Part II, Rev. Mat. Ibero. 1:1 (1985), pp. 145-201 \& 1:2 (1985), pp. 45-121.

[16] J. QING, On singularities of the heat flow for harmonic mapping from surface into spheres, Comm. Anal. Geom., 3 (1995), pp. 297-315.

[17] J. SACKS AND K. UHLENBECK, The existence of minimal immersion of two spheres, Ann. Math., 113 (1981), pp. 1-24.

[18] M. STRUWe, On the evolution of harmonic maps of Riemannian surface, Comm. Math. Helev., 60 (1985), pp. 558-581.

[19] M. StruWE, Large H-surfaces via the mountain-pass-lemma, Math. Ann., 270 (1985), pp. 441-459.

[20] C. Y. WANG, Bubble phenomena of certain Palais-Smale sequences from surfaces to general targets, Houston. J. Math., 22 (1996), pp. 559-590.

[21] G. F. WANG, Harmonic maps from a closed surface of higher genus to the unit 2-sphere, Internat. J. Math., 4 (1993), pp. 359-365.

[22] H. Wente, An existence theorem for surfaces of constant mean curvature, J. Math. Anal. Appl., 26 (1969), pp. 318-344.

[23] K. UHLENBECK, Morse theory by pertubation methods with applications to harmonic maps, Trans. Amer. Math. Soc., 267 (1981), pp. 569-583. 